\title{
A field-deployable compound-specific isotope analyzer based on quantum cascade laser and hollow waveguide
}

Sheng Wu, Andrei Deev

Sheng Wu, Andrei Deev, "A field-deployable compound-specific isotope analyzer based on quantum cascade laser and hollow waveguide," Proc. SPIE 8631, Quantum Sensing and Nanophotonic Devices X, 86310D (4 February 2013); doi: 10.1117/12.2003261

SPIE. Event: SPIE OPTO, 2013, San Francisco, California, United States 


\title{
A Field Deployable Compound Specific Isotope Analyzer based on Quantum Cascade Laser and Hollow Waveguide
}

\author{
Sheng Wu, Andrei Deev \\ PEER Institute \\ 738 Arrow Grand Circle, Covina, CA 91722 \\ California Institute of Technology \\ 139-74 Caltech, Pasadena, CA 91125
}

\begin{abstract}
A field deployable Compound Specific Isotope Analyzer (CSIA) coupled with capillary chromatogrpahy based on Quantum Cascade (QC) lasers and Hollow Waveguide (HWG) with precision and chemical resolution matching mature Mass Spectroscopy has been achieved in our laboratory. The system could realize 0.3 per mil accuracy for $12 \mathrm{C} / 13 \mathrm{C}$ for a Gas Chromatography (GC) peak lasting as short as 5 seconds with carbon molar concentration in the GC peak less than $0.5 \%$. Spectroscopic advantages of HWG when working with QC lasers, i.e. single mode transmission, noiseless measurement and small sample volume, are compared with traditional free space and multipass spectroscopy methods.
\end{abstract}

\section{KEY WORD LIST}

green photonics, quantum cascade laser; laser spectroscopy, Hollow Waveguide; Compound Specific Isotope Analyzer

\section{INTRODUCTION}

The current workhorse in stable isotope analysis is Isotope Ratio Mass Spectrometers (IRMS). Especially when integrated with Chromatography, the online GC-IRMS[1] or LC-IRMS have become the most powerful and necessary tool for scientists and have opened certain commercial market in geological survey and fossil fuel exploration. However, the weight, size and delicate nature of IRMS preclude field deployment. One of the disadvantages for IRMS is the requirement of constant "calibration standard"; this "calibration standard" for current isotope measurement is an analogy to "King's Foot for length measurement" centuries ago. The international carbon isotope standard, Pee Dee Belemnite (PDB), finds its origin in a fossil stone from South Carolina which was used as a door block at the University of Chicago and it was chosen by the founding pioneers in isotope chemistry, e.g. Harold Urey, as the first carbon isotope standard (perfect analogy of "King's Foot" for C isotope standard). This fossil stone had been used up long time ago and replaced with a secondary standard (replica of the "King's foot" for C isotope standard). The reason for the requirement of "King's Foot" is because of the nature of MS, i.e. the mass ion signal is an emission signal which strongly depends on the conditions of high vacuum, ionization (related to power quality) and vibrations, and thus constant calibration is required. Thus, even after many years of development IRMS is still remote for field deployment, not to mention airborne.

Optical absorption spectroscopy based Isotope Ratio analyzers, on the other hand, could be relatively stable, and inherently the fluctuations in background or baseline could be calibrated with a reference path, or blank sample measurement. Cavity enhanced absorption techniques such as Cavity-Ring-down-spectroscopy (CRDS) and Integrated Cavity Output Spectroscopy (ICOS), have given birth to several commercial optical isotope analyzers. These commercial isotope analyzers require much less frequent calibration than required by IRMS, and they don't need high vacuum and are much less demanding on power source quality, thus making them quite amenable for field deployment. However, these techniques have large sample cells and require temperature and pressure control and as we analyzed in detail in the sections below, their weight and power consumption could not be minimized; further,

Quantum Sensing and Nanophotonic Devices X, edited by Manijeh Razeghi, Eric Tournié, Gail J. Brown, Proc. of SPIE Vol. 8631, 86310D · C 2013 SPIE · CCC code: 0277-786X/13/\$18 · doi: 10.1117/12.2003261 
although calibration with "King's Foot" is no longer as necessary as IRMS, they still need routine calibration if higher level of accuracy is required, i.e. $<0.3$ permil over a period of several days[2]. The large sample cell also precludes them from being integrated with online high resolution capillary chromatography, thus limit these instruments to just a couple of simple chemical species --- IRMS still rules the bench[3]. There is also Photoacoustic spectroscopy (PAS) which could potentially have very small sample volume as well as footprint and weight, e.g. the Quartz Enhanced PhotoAcoutisc Spectroscopy (QEPAS)[4], while delivery high sensitivities without need for expensive detectors. But PAS in general need to operate at close to one atmosphere pressure for optimum sensitivity, and therefore the linewidth is limited to $0.1 \mathrm{~cm}^{-1}$, and for accurate isotope ratio measurement, this limit means incomplete separation of interfering isotopologue or other gas species, causing inaccurate measurement. The emission nature of PAS signal, i.e. the signal strongly depends on sample conditions such as moisture, also means calibration with a "King's Foot" standard might be much more often than Beer's law based absorption spectroscopy techniques.

\section{Reviving Infrared detector for Gas Chromatography with Quantum Cascade Lasers}

Before Mass Spectroscopy (MS) became the benchmark instrument for Chromatography in the late 70's and throughout today, Infrared (IR) spectroscopy had enjoyed a short period of being the solo detector available for analytical chemists which could help distinguish chemicals for chromatography. But, as soon as MS became available, chromatography also went a transformation from packed column to capillary columns, thus the tiny sample volume of newer capillary Chromatography strongly favor MS over IR whose large sample volumes crippled its performance severely.

One limit for the IR's sample volume when operating with GC (Gas Chromatography) is the fact that IR light from blackbody sources could not propagate through narrow bore hollow waveguide (HWG) which also passes chemical elutes from capillary columns[5]. We are the first to report that Quantum Cascade (QC) lasers could be efficiently coupled into long HWG with same internal diameters as current GC capillaries. Thus, the sensitivity of online GC-IR is improved about 3 orders of magnitude and now comparable with GC-MS. We have conducted intensive research and developed a GC-IR2 (Infrared Isotope Ratio) system that could deliver similar accuracy as the GC-IRMS, the Crown Jewel of MS in terms of accuracy; and most important this GC-IR2 could be field deployed. As we discussed in detail in the sections below, our GC-IR2's small sample volume makes sample refreshing faster and compatible with high resolution GC; it also uses very light vacuum system thus consuming very little power.

\section{EXPERIMENT}

\section{How could HWG + QCL sensor achieve high accuracy?}

For absorption spectroscopy, conventional wisdoms often treat HWG as incapable of higher accuracy measurement because majority of us spectroscopists [5-7], including the authors here 5 years ago, believe that HWG supports multimode inherently. However, work in references [6, 7] excited HWG's higher order modes and HWG is not maintained to filter out the higher order modes, and thus noise due to multimode detection is present and needs to be alleviated.

Our latest $R \& D$ results prove that for Mid-Infrared lasers with wavelength larger than 3 microns, it is easily to filter out higher order modes and keep only single mode output with HWG tube, and then maintain single mode inside the HWG sample cell. This way, it is easy to achieve high accuracy inside hollow waveguide with QC lasers, i.e. 10${ }^{6} / \mathrm{Hz}^{1 / 2}$, similar to free space single pass spectroscopy. This result is similar to the finding by PNNL group back in 2002[8] when they used a near-IR diode to achieve similar results which is much more difficult and different than mid-IR wavelength lasers in terms of wavelength/HWG bore size mismatch. Their preliminary results indicate that the measurement accuracy of HWG with infrared lasers could be much better than multipass cell, close to free space single pass beam measurement. Currently, the multipass cell could often demonstrate measurement accuracy better than $10^{-5} / \mathrm{Hz}^{1 / 2}$; and our work and PNNL's work in 2002 demonstrate that HWG infrared lasers could achieve similar or better accuracy. 


\section{Single mode filtering and propagation of QCL inside HWG}

Currently the most popular HWGs are silica capillaries with inside coatings. The coatings come in two types. The first kind of HWG is coated with dielectric only, the variation of thickness and layers of coating created the photonic crystal structure that is similar to regular dielectric coatings on reflective mirrors; this type of HWG has selective limited reflective band that cover part of the Mid-IR region. The second type of HWG is coated with metal-dielectric film, and the reflective band could be very large, almost covering the complete Mid-IR region although the reflectivity is not ideal or optimized, e.g. Polymicro's HWEA series HWG. The beam diameter (taken as $70 \%$ of the capillary diameter) and divergence product of this type of HWG is usually $6 \sim 10$ times the diffraction limit of the wavelength in the Mid-IR for capillary inside diameter less than $1 \mathrm{~mm}$. The mode output from larger bore HWG (e.g. $>500 \mu \mathrm{m}$ ) also likes to become multimode if the HWG is bent[9]; although the multimode effect due to bending will be reduced for small bore HWG, e.g. ID less than 20 times the wavelength, and such small bore HWG could even filter out input with higher order modes if small bore HWG sufficiently long is used[9, 10]. Such filtering effect is already used to filter out higher order mode, and ensure tight focusing of single mode output from a QCL laser in Quartz Enhanced Photo Acoustic Spectroscopy[11]. However, HWG with such mode filtering capability usually runs over a meter, making the system bulky and unstable if the long HWG is disturbed during operation.

We found that small short piece of simple HWG could conduct perfect mode filtering when properly used. We achieved a single mode output at only 1.5 times diffraction limit with a simple filter HWG of $6 \mathrm{~cm}$ length.

\section{The weight and power consumption of the vacuum system goes up faster than the dead volume of the absorption cell}

In the case of $\underline{H W G}$ sample cell, the cell volume is only $1 \mathrm{ml}$ for a 1.2 meter long and $1 \mathrm{~mm}$ diameter HWG. The pressure could be maintained at $\sim 60$ Torr at a flow rate of $6 \mathrm{ml} / \mathrm{min}$, based on the note below on "calculation of pressure drop in small bore HWG at a given flow rate", the pressure drop is $\sim 10$ Torr when the initial pressure is at 60Torr.

The dead volume is thus only $0.1 \mathrm{ml}(\mathrm{STP})$ and could maintain $1 \mathrm{~Hz}$ sample refresh rate at a flow rate of $6 \mathrm{ml} / \mathrm{min}$. At $6 \mathrm{ml} /$ minute (STP) flow rate, a pair of commercial-off-the-shelf micro diaphragm vacuum pumps (Parker Hargraves CTS micro diaphragm pump weighs less than 50grams each in half a baseball size) in series could maintain $<50$ Torr vacuum (flow is limited to $<10 \mathrm{cc} /$ minute STP but enough for our HWG sample cell at $1 \mathrm{~Hz}$ sample refreshing rate) with a weight less than 100grams, and power consumption less than 3W (9V @ $<150 \mathrm{~mA}$ for each pc)!

\section{Note: calculation of pressure drop in small bore HWG at a given flow rate}

The Pressure Drop product (PDP), i.e. $p_{1} \delta p$, is essentially proportional to the $\mu \mathrm{L} / \mathrm{D}^{4}$, and also ambient flow rate $\mathrm{Q}$ (with unit of $\mathrm{Pa}^{*} \mathrm{~m}^{3} / \mathrm{sec}$ ), i.e.

$\mathrm{p}_{1} \delta \mathrm{p}=8.31 \mu * \mathrm{~T} * 128 / \pi * \mathrm{Q}_{\text {nor }} * \mathrm{~L} / \mathrm{D}^{4}$

$\mathrm{Q}_{\text {nor }}$ here has unit of mole/sec (or equivalent to $2,240 \mathrm{~Pa}^{*} \mathrm{~m}^{3} / \mathrm{sec}$ ), $\mathrm{p}, \delta \mathrm{p}$ have unit of $\mathrm{Pa}$, and $\mathrm{L}$ and $\mathrm{D}$ are the length and diameter of the HWG cell and have unit of meter, and T is temperature in Kelvin, and $\mu$ is dynamic viscosity and has unit of $\mathrm{Pa}^{*} \mathrm{Sec}$. Based on this relation we know that the refreshing rate of a HWG cell operating under low pressure conditions could not be increased without limit as opposed to conventional wisdom, but it is still much faster than other absorption cells, as we demonstrated in our GC coupled Isotope Ratio analyzer based on HWG and QC lasers.

\section{Examples of other absorption cell based sensor platform}

In the case of $\underline{C R D S}$, it has the smallest sample cell volume possible, usually less than $100 \mathrm{ml}$, but still larger than $20 \mathrm{ml}$ ( $1 \mathrm{ft}$ length and $3 / 8$ " clear aperture) even with minimal volume design. If we want to update the sample at $1 \mathrm{~Hz}$ rate, the pump has to maintain vacuum pressure, i.e. typical $100 \mathrm{mBar}$, at a flow rate $\sim 240 \mathrm{ml} / \mathrm{min}$ (STP), i.e. $60 \mathrm{sec} \mathrm{x}$ 
$20 \mathrm{ml} / \mathrm{sec} \times 100 \mathrm{mBar} \times 2$ (a factor of 2 is needed to refresh the cavity because it is not a simple straight tube for sample flow). At this flow rate, the system has to use a diaphragm pump large enough that well over $1 \mathrm{~kg}$ dry weight is warranted, not to mention that extra power supply needed to power such a pump.

In the case of ICOS and multi-pass cells, their sample cell volume is usually much larger than $100 \mathrm{ml}$, thus, much larger vacuum pump is needed to refresh the sample at $1 \mathrm{~Hz}$ rate, usually the weight of such pump alone is much larger than $5 \mathrm{~kg}[12]$, not to mention the power needed for such pumps is usually close to $1 \mathrm{~kW}$. The result in the latest paper on a UAV GHG analyzer[13] also confirms our analysis here, their OA-ICOS system (400ml cell) uses a pump which only refreshes the sample every 9 seconds, and already it weighs close to $1 \mathrm{~kg}$, and is the main power drain in their total system which consumes only $70 \mathrm{~W}$. Also, at sample flow rate goes much faster, the speed of fresh sample inside the sample cell will be quite high that the thermal equilibrium might not be reached inside large cells. For small bore cell like HWG, this concern is much relaxed.

We first demonstrated and reported in literature that the coupling efficiency is over $50 \%$ for semiconductor epi lasers, e.g. QC lasers, into HW due to QC laser's high brightness[14]. The reason for such high efficiency is the brightness or brilliance of semiconductor lasers --- the same property that enabled killer application platforms for diode lasers, such as DVD and fiber optical communications. We are also the first to demonstrate coupling HWG to capillary GC and QC laser to conduct online high precision Infrared spectroscopy, and especially for compound specific stable isotope ratio analysis[14].

In the past 3 years, we have developed the online $\mathrm{CO}_{2}$ Infrared Isotope Ratio (IR2) sensor module for $\mathrm{GC}$ using QC laser and Hollow Waveguide; we have succeeded in using a CW QC laser for simultaneously measuring ${ }^{12} \mathrm{CO} 2$ and ${ }^{13} \mathrm{CO} 2$ at $\sim 2,299.70 \mathrm{~cm}^{-1}$. The instrument is now able to measure $\delta^{13} \mathrm{C}$ with an error within \pm 0.2 per mil.

\section{RESULTS}

To achieve such performance, we have to conduct absorption spectroscopy under lightly vacuumed lower pressure conditions, i.e. $<60$ Torr, where pressure broadening is minimized to the scale of Doppler broadening. The linewidth we achieved, i.e. $\sim 0.005 \mathrm{~cm}^{-1}$, allow us to measure only one kind of the isotopologue while minimizing interferences from other gases or species. We first theoretically simulated the pressure changed inside a $1 \mathrm{~mm}$ diameter HWG under low pressure conditions as we discussed in section about the our proposed method above. Then, we experimentally verified the relationship of flow rate, pressure drops inside the $1 \mathrm{~mm}$ bore size HWG over 1meter, i.e. we could achieve homogenous pressure, i.e. $<5 \%$ difference, in the 1 meter long hollow waveguide when it was pumped down to low vacuum, e.g. 50 Torr, with a typical capillary flow of $3 \mathrm{cc} /$ minute (STP) and backed by a pair of CTS micro diaphragm pump from Hargraves. The linear flow speed is about $1.2 \mathrm{~meter} / \mathrm{second}$, and this allows $4 \mathrm{~Hz}$ sample rate for our GC-IR2 product which uses a $30 \mathrm{~cm}$ long $\mathrm{HWG}$; at the same time, the flow rate is compatible with capillary GCs. The optical train layout of our IR2 module is given in figure 1.

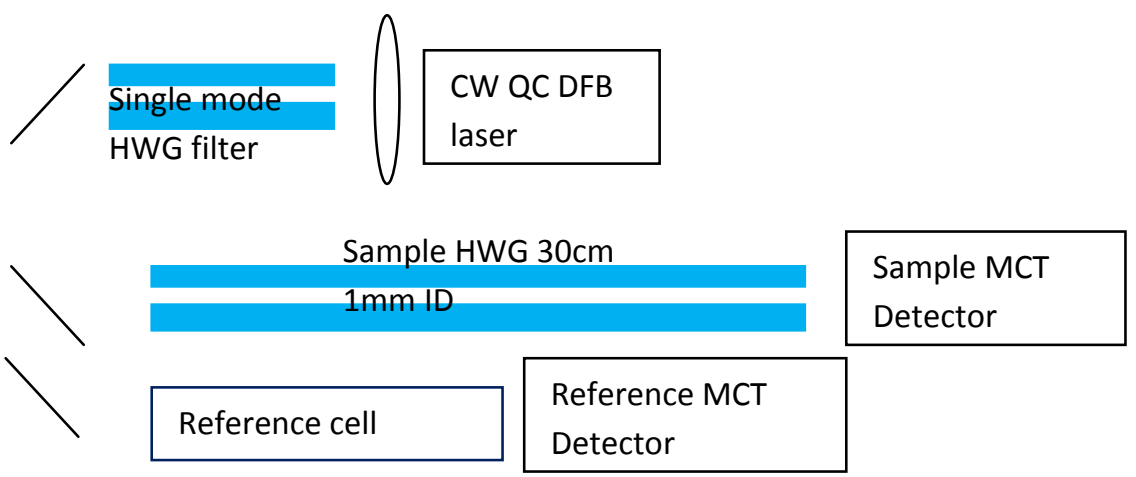

Figure 1. Optical train layout of our IR2 (Infrared Isotope Ratio) analyzer module 
Briefly, we use a short piece of single mode HWG to filter out the higher order mode of the QC DFB laser output, and the close to diffraction limit output is split into the sample and reference channels. The sample beam goes through the $30 \mathrm{~cm}$ long $1 \mathrm{~mm}$ diameter sample $H W G$, and the reference beam goes through another $10 \mathrm{~cm}$ long reference cell before both beams were detected with two detectors. The signals are collected with a National Instrument PCI-6110E card which also provides the customized current ramp function for modulating the laser wavelength at $5 \mathrm{kHz}$. The sample HWG is temperature stabilized at $40 \pm 0.005^{\circ} \mathrm{C}$ range. Figure 2 shows the spectra fitting window of our GC-IR software, it is acquiring data from the IR2 (Infrared Isotope Ratio) module, the current was at zero before the ramp, thus creating a background for signal ground reference, and each ramp scans $\sim 1 \mathrm{~cm}^{-1}$. Here, we are fitting the lines at 2,299.5 2,299.80 $\mathrm{cm}^{-1}$, calculating the ratio of ${ }^{13} \mathrm{C}^{12} \mathrm{C}$ by measuring and fitting the ${ }^{12} \mathrm{CO}_{2}$ line at $2,299.64 \mathrm{~cm}^{-1}(0.1605 \mathrm{msec})$ and ${ }^{13} \mathrm{CO}_{2}$ line at $2,299.80 \mathrm{~cm}^{-1} \cdot(0.1735 \mathrm{msec})$. The $3{ }^{\text {rd }}$ smaller peak $(0.1510 \mathrm{msec})$ is another hot band line for ${ }^{12} \mathrm{CO}_{2}$.

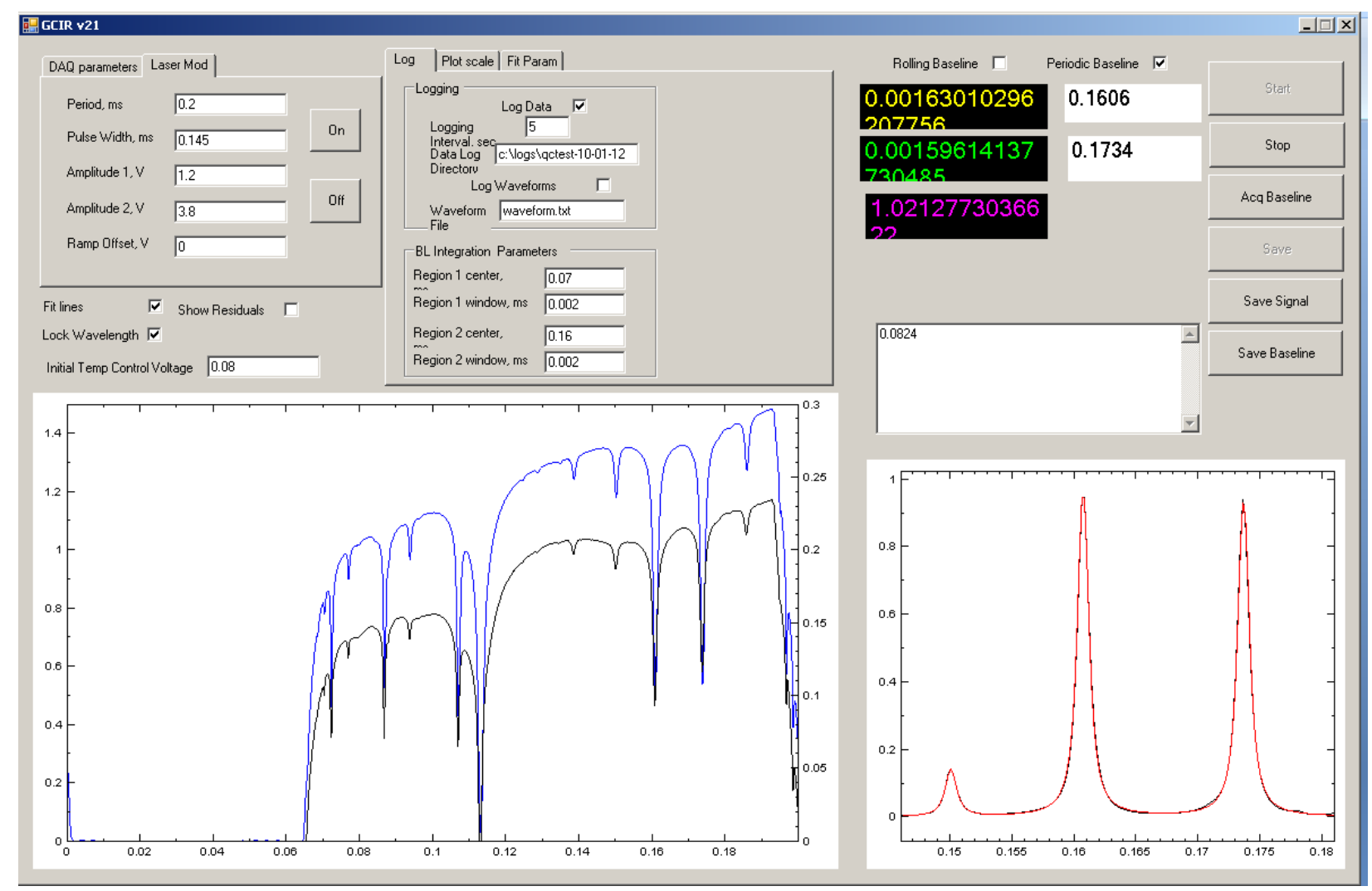

Figure 2. GC-IR2 software shows the fitted spectra for $1 \% \mathrm{CO}_{2}$ in sample $\mathrm{HWG} \&$ reference cell.

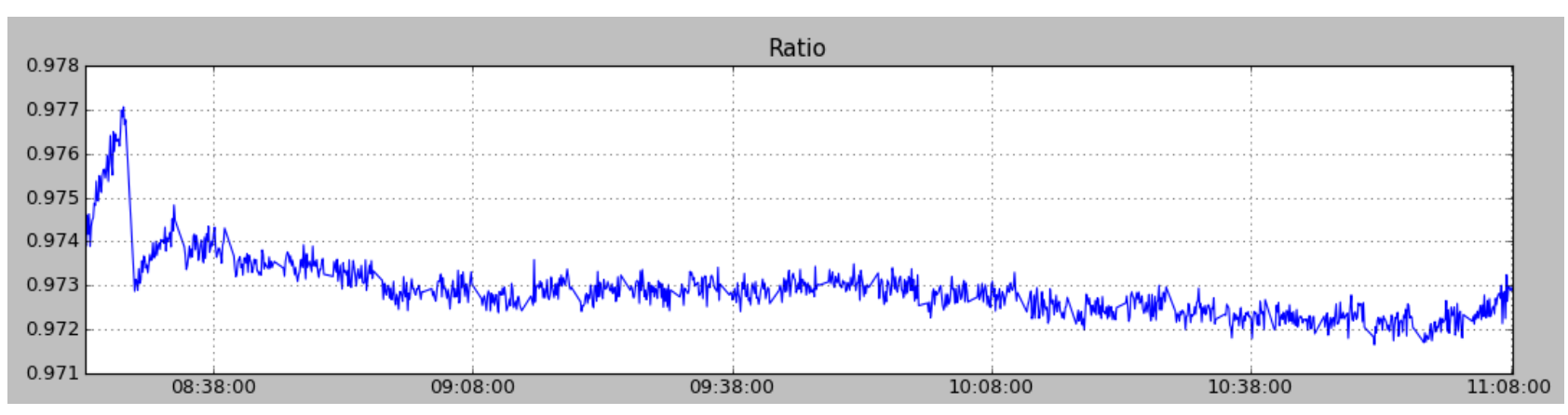


Figure 3. Logged ratio (arbitrary before calibrate to standard which is close to 1) after system box is closed. X-axis is time (hr:min:sec) and $\mathrm{Y}$-axis is the ratio of the two peaks.

Figure 3 shows the logged ratio (arbitrary, before scaled to standard) result of a continuous flow $0.2 \% \mathrm{CO} 2$ in the $30 \mathrm{~cm}$ long cell. We could see after we turn on the system, there is a 20 minutes warm up time, and 3 hours after that the drift is less than 1 per mil, while short term noise, $\delta^{13} \mathrm{C}$, is under $0.2 \mathrm{per} \mathrm{mil} / \mathrm{Hz}^{1 / 2}$. Again, we could achieve such high accuracy because the beam entering the sample HWG is single mode and maintained single mode.

We then integrated our IR2 module online with a GC system where the hydrocarbons are separated, combusted into $\mathrm{CO}_{2}$ and feed directly into our IR2 for analysis. This is another challenge because here the GC peak has fast changing mixing ratio from $<10 \mathrm{ppm}$ to several percent, and our IR2 is calibrated with different concentrations in order to get a real integrated value for the GC elute peaks. The latest results show that we are getting $\delta^{13} \mathrm{C}<0.3$ per mil for propane, ethane and methane when we injected the mixtures into the GC-IR2 system. The large dynamic range of our system, i.e. usable absorption ranging from $<2 \%$ to over $60 \%$ makes our instrument quite flexible, and also means our proposed real measurement of temperature is quite possible even when the peaks involved has a factor of 10 difference in absorptions. From our results presented above, we could see that the results are scalable for 1.2 meter pathlength for the proposed airborne system, i.e. provide sampling rate at $1 \mathrm{~Hz}$, and reach the standard accuracy for atmospheric $\mathrm{CO}_{2}$.

\section{DISCUSSIONS}

\section{Advantages and disadvantages of the IR2 sensor}

The IR2 sensor owes its advantages to the inherent low dead volume and reasonable pathlength, and moreover, the near diffraction limit beam size and divergence product enables high accuracy measurement. With the recent revival of Mid-IR spectroscopy due to QC lasers and detectors, these inherent properties could improve the IR2 sensor accuracy and speed significantly. Here, we have demonstrated that by operating HWG with a reasonable inside diameter and under light vacuum, the IR2 sensor could give both high accuracy and high spectral resolution, i.e. Doppler broadening limit. The result is that the IR2 detector could now be couple to GC line and work as an online chemical specific detector. The accuracy could match that of MS, and has the potential advantage of achieving a calibration free operation.

However, the pathlength of the the IR sensor is still limited. This is due to the nature that limited number of passes of HWG, i.e. 1 to 2 passes. Therefore for relatively weaker IR absorbing chemicals, IR2 is not sensitive enough to provide the kind of accuracy to compete to MS yet. We propose and demonstrate that Whispering Gallery Mode could be the next technology that could effectively extend the pathlength and at the same time further reducing the dead volume[15].

\section{CONCLUSION}

Here, we first give an overview of the current status of compound specific isotope analyzers, as well as the current general state of the chemical specific detectors, i.e. Mass Spectrometer (MS) versus Infrared (IR). Then we discussed the necessary improvements for IR to compete with MS again and how we use QC laser and HWG to achieve such performances by ensuring single mode coupling and light vacuum operation. Results are presented and demonstrate that we could achieve 0.3 per mil accuracy for online GC compound specific carbon isotope analysis, and most important, the IR2 sensor has the potential to achieve calibration free operation. As the performance of QC laser improves in terms of tunability and operation conditions, the future bodes well for IR as an online detector for GC as well as other micro and fast analysis. 


\section{ACKNOWLEDGEMENT}

We gratefully acknowledge the financial support from DOE-RPSEA project titled 'Novel Gas Isotope Interpretation Tools to Optimize Gas Shale Production' (DE-AC26- 07NT42677).

\section{REFERENCES}

1. Sessions, A.L., Isotope-ratio detection for gas chromatography. Journal of Separation Science, 2006. 29: p. 1946-1961.

2. Rella, C., Reduced drift, high accuracy stable carbon isotope ratio measurements using a reference gas with the Picarro CO2 G2101-i gas analyzer. Picarro White Paper. http://www.picarro.com/assets/docs/White Paper G2101-i Drift Reduction.pdf.

3. Zare, R.N., et al., High-precision optical measurements of C-13/C-12 isotope ratios in organic compounds at natural abundance. Proceedings of the National Academy of Sciences of the United States of America, 2009. 106(27): p. 10928-10932.

4. Dong, L., et al., QEPAS spectrophones: design, optimization, and performance. Applied Physics BLasers and Optics, 2010. 100(3): p. 627-635.

5. Griffith, P.R. and J.A. de Haseth, Fourier Transform Infrared Spectrometer. Chemical Analysis: A series of Monographs on Analytical Chemistry and Its Applications, ed. J.D. Winefordner. 2007: Wiley Interscience.

6. Chen, J., et al., Resolution limits of laser spectroscopic absorption measurements with hollow glass waveguides. Appl. Opt., 2010. 49(28): p. 5254-5261.

7. Chen, J., et al., Low-level and ultralow-volume hollow waveguide based carbon monoxide sensora. Optics Letters, 2010. 35(21): p. 3577-3579.

8. Blake, T.A., et al. Absorption spectroscopy in hollow-glass waveguides using infrared diode lasers. in Diode Lasers and Applications in Atmospheric Sensing. 2002. Seattle, WA, USA: SPIE.

9. Kriesel, J.M., et al., Hollow core fiber optics for mid-wave and long-wave infrared spectroscopy. 2011: p. 80180V-80180V.

10. Harrington, J.A., C.M. Bledt, and J.M. Kriesel, Hollow waveguides for the transmission of quantum cascade laser (QCL) energy for spectroscopic applications. 2011: p. 789414-789414.

11. Spagnolo, V., et al., Part-per-trillion level detection of SF6 using a single-mode fiber-coupled quantum cascade laser and a quartz enhanced photoacoustic sensor SPIE Photonics West Conference, Nano and Quantum Sensing, 2013, 2013: p. Paper 8631-32.

12. Shorter, J.H., et al., Multicomponent Breath Analysis With Infrared Absorption Using RoomTemperature Quantum Cascade Lasers. leee Sensors Journal, 2010. 10(1): p. 76-84.

13. Berman, E.S.F., et al., Greenhouse gas analyzer for measurements of carbon dioxide, methane, and water vapor aboard an unmanned aerial vehicle. Sensors and Actuators B: Chemical, 2012. 169(0): p. 128-135.

14. Wu, S., et al., Hollow waveguide quantum cascade laser spectrometer as an online microliter sensor for gas chromatography. Journal of Chromatography A, 2008. 1188(2): p. 327-330.

15. Wu, S. and A. Deev, Observation of whispering gallery modes in the mid-Infrared with a Quantum Cascade Laser: possible applications to nanoliter chemical sensing. SPIE proceedings, Paper 722260 Photonics West 2009, 2009. 